\title{
Seguridad y eficiencia de sedación balanceada con propofol y remifentanil en endoscopia digestiva alta diagnóstica. Una experiencia exitosa
}

\section{A Successful Experience: Safety and Efficiency of Balanced Sedation with Propofol and Remifentanil in Diagnostic Endoscopy}

\author{
Camilo Blanco A., MD, MSc Edu, ${ }^{1}$ Karen Russi G., MD, ${ }^{2}$ Diana Chimbi, Enf, ${ }^{3}$ Alberto Molano A., MD, ${ }^{4}$ Alix Forero, MSc Edu. ${ }^{5}$
}

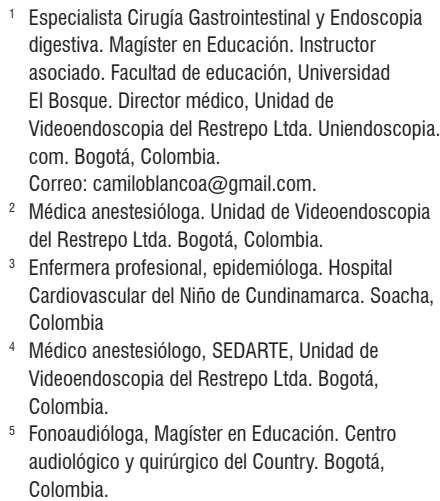

5 Fonoaudióloga, Magíster en Educación. Centro audiológico y quirúrgico del Country. Bogotá, Colombia.

\begin{abstract}
Resumen
La sedación es una técnica anestésica de amplio uso en los procedimientos endoscópicos digestivos actuales dado su claro beneficio en la tolerancia y comodidad para el paciente y el endoscopista. El medicamento de mayor uso en la actualidad para utilizarse como monosedación es el propofol, pero los esquemas balanceados utilizando más de un medicamento ahora son ampliamente usados en endoscopia diagnóstica o terapéutica. La sedación balanceada utilizando propofol y remifentanilo permite la potenciación sinérgica de un sedante con un opioide de ultracorta acción, lo que a su vez favorece la disminución respectiva de cada dosis. Se presenta una serie de 1148 pacientes llevados a endoscopia digestiva alta diagnóstica con dosis promedio de remifentanilo de $0,9 \mu \mathrm{g} / \mathrm{kg}$ de peso y de propofol de $0,47 \mathrm{mg} / \mathrm{kg}$ de peso, sin eventos adversos graves, con excelente satisfacción para el endoscopista y con muy bajo costo de la dosis por medicamento, con lo que se infiere que es un esquema seguro y eficiente.
\end{abstract}

\section{Palabras clave}

Sedación, endoscopia digestiva alta diagnóstica, propofol, remifentanilo, sedación balanceada.

\begin{abstract}
Sedation is an anesthetic technique that is widely used in current digestive endoscopic procedures because of its clear benefits for patients' tolerance and comfort and for the endoscopist. Propofol is the most commonly used drug in monosedation, but balanced regimens using more than one drug are now widely used in diagnostic and therapeutic endoscopy. Balanced sedation using Propofol and Remifentanil allows synergistic potentiation of a sedative with an ultra-short acting opioid which in turn favors decreases of each dose. This is a series of 1,148 patients who underwent diagnostic endoscopy under balanced sedation with average Remifentanil doses of $0.9 \mathrm{mcg} / \mathrm{kg}$ of body weight and average Propofol doses of $0.47 \mathrm{mg} / \mathrm{kg}$ of body weight. There were no serious adverse events, endoscopists were highly satisfied with the procedures, and costs per drug dose were very low. This is clearly a safe and efficient scheme.
\end{abstract}

Keywords

Sedation, diagnostic endoscopy, propofol, remifentanil, balanced sedation.

\section{INTRODUCCIÓN}

La sedación es la alteración farmacológicamente controlada del estado de conciencia de un paciente, que permite reali- zar procedimientos que pueden ser molestos o dolorosos, estado que puede oscilar desde ansiólisis hasta anestesia general $(1,2)$. El nivel de dicha depresión es un contínuo (no absolutamente controlable, mas sí prevenible) de llegar 
a los niveles más profundos si se utilizan fármacos específicos, en dosis adecuadas y pertinentes para la complejidad del procedimiento a realizar (3).

En endoscopia digestiva diagnóstica, son objetivos principales de la sedación el garantizar la tranquilidad, comodidad y cooperación del paciente a lo largo del procedimiento. Otros objetivos secundarios apuntan a generar amnesia, disminuir o quitar las náuseas e incluso a hacer un control efectivo del dolor asociado con algunas intervenciones (4).

Dentro del proceso de calidad global en la atención a pacientes, se considera que la sedación aporta a una mayor precisión y exactitud de los exámenes con una disminución del temor asociado con su realización; pero debe, a su vez, proporcionar seguridad, entendida como la prevención, control y manejo de complicaciones inherentes a su administración tales como hipoxia, hipotensión, bradicardia, tórax en leño, reacciones alérgicas, intubación orotraqueal, hospitalización no programada e incluso la muerte (5).

Iniciamos nuestra experiencia en sedación en el año 2002, sabiendo la posibilidad de diferentes esquemas para diferentes tipos de procedimientos endoscópicos. Con la participación de un anestesiólogo, utilizamos varios esquemas para endoscopia digestiva alta y baja diagnóstica (con medicamento único, doble o triple medicamento; con midazolam, fentanilo, propofol, remifentanilo o ketamina), impulsados por el concepto de eficiencia en la práctica, entendida como la disminución en el gasto de atención y menor tiempo necesario para recuperación.

La sedación balanceada se entiende como el uso de más de un agente anestésico, sedante o analgésico, en una proporción que garantice ansiólisis, sedación, analgesia y amnesia $(7,8)$. Presentamos nuestra experiencia exitosa, sin eventos adversos significativos, de una serie seleccionada de 1148 pacientes llevados a endoscopia digestiva alta diagnóstica, realizada por un solo endoscopista (Camilo Blanco), con esquema de sedación balanceada administrada por un solo anestesiólogo (Karen Russi), con 2 medicamentos (propofol-Profol ${ }^{\odot} 1 \% 10 \mathrm{mg} / \mathrm{mL}$ y remifentanilo Ultiva ${ }^{\odot} 2 \mathrm{mg} 20 \mu \mathrm{g} / \mathrm{mL}$ ), para lograr niveles de sedación II y III (ansiólisis y sedación consciente) de acuerdo con la Tabla 1 de clasificación (4).

\section{MATERIALES Y MÉTODOS}

\section{Tipo de estudio}

Estudio descriptivo retrospectivo realizado en la Unidad de Videoendoscopia del Restrepo Ltda., institución prestadora de salud (IPS) ubicada en el centro sur de la ciudad de Bogotá, en pacientes llevados a endoscopia digestiva alta diagnóstica durante el período comprendido entre mayo de 2013 y noviembre de 2014. Ya que no era un estudio experimental, no requirió consentimiento para la inclusión de los pacientes en la base de datos y el estudio fue aprobado por el comité de ética de la unidad.

\section{Criterios de inclusión}

Con la aprobación institucional, se registraron 1148 pacientes consecutivos atendidos por un solo especialista en cirugía gastrointestinal y endoscopia digestiva (CB) y una sola de las anestesiólogas del grupo de trabajo (KR). Los pacientes incluidos no tenían comorbilidades cardíacas, pulmonares o metabólicas significativas, y de acuerdo con la Sociedad Americana de Anestesiología (ASA) estuvieron clasificados como I y II (9).

Todos los pacientes autorizaron mediante consentimiento informado la realización del procedimiento, bajo sedación. Para esta serie, solo se tuvo en cuenta a los pacientes atendidos los días martes y sábado de la semana, en las fechas en que coincidían los 2 investigadores principales.

\section{Criterios de exclusión}

Institucionalmente no se atienden pacientes en niveles ASA III o IV. También fueron excluidos pacientes con aler-

Tabla 1. Clasificación de niveles de sedación

\begin{tabular}{|c|c|c|c|}
\hline \multicolumn{2}{|r|}{ Niveles de sedación } & \multirow[t]{2}{*}{ Estatus del paciente } & \multirow{2}{*}{$\begin{array}{l}\text { Eventos potenciales } \\
\text { Excitación, agitación, retiro del } \\
\text { endoscopio }\end{array}$} \\
\hline I & Despierto, alerta & & \\
\hline II & Sedación ligera & Colaborador, ojos abiertos, respiración espontánea, respuesta a estímulos verbales & \\
\hline III & Sedación moderada & Somnoliento, ojos cerrados, responde a estímulos táctiles & \\
\hline IV & Sedación profunda & $\begin{array}{l}\text { Pobre respuesta a estímulos vigorosos, hipotonía muscular y pérdida de reflejos, sin } \\
\text { ventilación espontánea, estabilidad cardiovascular }\end{array}$ & Hipoventilación, hipoxia \\
\hline V & Anestesia general & Supresión de reflejos respiratorio y cardiovascular, obstrucción de vía aérea & Apnea, paro cardiorrespiratorio \\
\hline
\end{tabular}

Adaptada de: Lightdale JR. Endoscopia gastroenterológica. 2.a edición. Venezuela: Amolca; 2012. p. 57-65. 
gia conocida a los medicamentos utilizados, al huevo o a la soya, y pacientes que rechazaron la sedación.

\section{Objetivo}

El objetivo en la serie fue analizar y mostrar la seguridad y la eficiencia del sistema de sedación balanceada usando propofol y remifentanilo en endoscopias digestivas altas diagnósticas, en un escenario ambulatorio de agenda abierta. La seguridad se determinó por la ausencia de eventos adversos asociados con la sedación (hipoxia, hipotensión, bradicardia, tórax en leño, reacciones alérgicas, intubación orotraqueal, hospitalización no programada y muerte).

La eficiencia se planteó al calcular el costo por paciente del propofol y remifentanilo usados en el sistema balanceado, de acuerdo con edad y peso corporal en $\mathrm{kg}$, y también al poder precisar la satisfacción de la sedación desde el punto de vista del endoscopista de acuerdo con una escala validada.

\section{Condiciones de atención}

Previa suplementación de oxígeno al 3\% con cánula nasal y monitorización electrocardiográfica y de pulsioximetría, se aseguró de no iniciar ningún procedimiento si la saturación de oxígeno $\left(\mathrm{Sat}_{2}\right)$ estaba por debajo de $90 \%$ y la presión arterial (PA) por debajo de $90 / 70 \mathrm{~mm} \mathrm{Hg}$ o por encima de $150 / 95 \mathrm{~mm} \mathrm{Hg}$. La mezcla balanceada fue administrada por la anestesióloga en bolos, con jeringas de 5 o $10 \mathrm{~mL}$, inyectando manualmente y a una velocidad aproximada de $5 \mathrm{~mL}$ en 30 segundos. La cantidad total de la mezcla calculada fue administrada, excepto que durante la infusión el paciente presentara tempranamente nistagmo vertical, tuviera una hipotonía muscular marcada o fuera evidente la supresión de su ventilación. Durante el procedimiento, el anestesiólogo o la enfermera asistente verificaban cada 15 segundos el nivel de respuesta del paciente a estímulos verbales.

Se consideró hipoxia que requirió intervención del anestesiólogo cuando la saturación del paciente estuvo por debajo del $90 \%$ por un período mayor a 30 segundos y que no corrigió con la hiperextensión de la mandíbula o el estímulo vigoroso. Se realizaría control de la vía aérea ante una persistencia de depresión respiratoria o de hipoxia no corregible con dispositivo de ventilación manual con presión positiva.

La endoscopia digestiva alta fue realizada de acuerdo con los parámetros de endoscopia sistemática descrita por Yao (10) y todas incluyeron una toma de biopsias de acuerdo con el sistema de Sidney (11).

El endoscopista, al final del examen, asignó un puntaje de satisfacción de acuerdo con la escala: procedimiento fácil para niveles de sedación II y III, procedimiento adecuado para el nivel de sedación IV, y procedimiento dificultoso para niveles de sedación I o V (12).
Terminado el procedimiento, los pacientes fueron trasladados a sala de recuperación donde estuvieron monitorizados y controlados por otro auxiliar de enfermería y fueron dados de alta con la autorización del anestesiólogo una vez cumplieran con un puntaje de 14 en la clasificación de la escala de Aldrete.

\section{Análisis estadístico}

La base de datos fue depurada con Excel y procesada en el programa estadístico Epi-Info. Se realizó un análisis univariado y multivariado. Los parámetros continuos fueron presentados como medidas de tendencia central y desviación estándar (DE).

\section{RESULTADOS}

Se tomó información de 1148 pacientes a quienes se les realizó endoscopia digestiva alta diagnóstica, realizadas en el período comprendido entre mayo de 2013 a noviembre de 2014, el 100\% de ellos bajo sedación balanceada con propofol y remifentanilo.

En la serie total, el 59\% fueron mujeres y el $41 \%$ fueron hombres, mientras que en el grupo de ASA II hubo una mayor proporción de género femenino (68\% frente al $32 \%)$. La media del peso para el grupo de pacientes ASA I fue de $64 \mathrm{~kg}$, para pacientes ASA II, $68 \mathrm{~kg}$ y para la serie total, $66 \mathrm{~kg}$ (Tabla 2).

Tabla 2. Características generales

\begin{tabular}{lcccccc}
\hline & \multicolumn{5}{c}{ ASA } \\
\cline { 2 - 7 } & \multicolumn{2}{c}{ I } & \multicolumn{2}{c}{ II } & Total \\
\hline Género & & & & & & \\
$\quad$ Femenino & 440 & $55 \%$ & 242 & $68 \%$ & 682 & $59 \%$ \\
$\quad$ Masculino & 353 & $45 \%$ & 113 & $32 \%$ & 466 & $41 \%$ \\
Total & 793 & $69 \%$ & 355 & $31 \%$ & 1148 & \\
Peso (kg) & & & & & & \\
$\quad$ Media & 64 & & 68 & & 66 \\
\hline
\end{tabular}

El 69\% (n=793) de los pacientes fueron clasificados como ASA I, estando el $83 \%$ de ellos en el grupo de edad entre 17 a 59 años. Solo un $4 \%$ fueron menores de 17 años, el $11 \%(n=90)$ estuvo entre 60 y 74 años y el $1 \%(n=11)$ fueron mayores de 75 años.

Por su parte, el 31\% ( $\mathrm{n}=355)$ de los pacientes fueron clasificados como ASA II y, diferente al grupo ASA I, solo el $51 \%$ estuvo entre 17 a 59 años; a su vez hubo un aumento del 35\% ( $n=123)$ de pacientes en el grupo de 60 a 74 años y al $14 \%(n=50)$ de los pacientes de mayores 75 o más años (Figura 1, Tabla 3 ). 


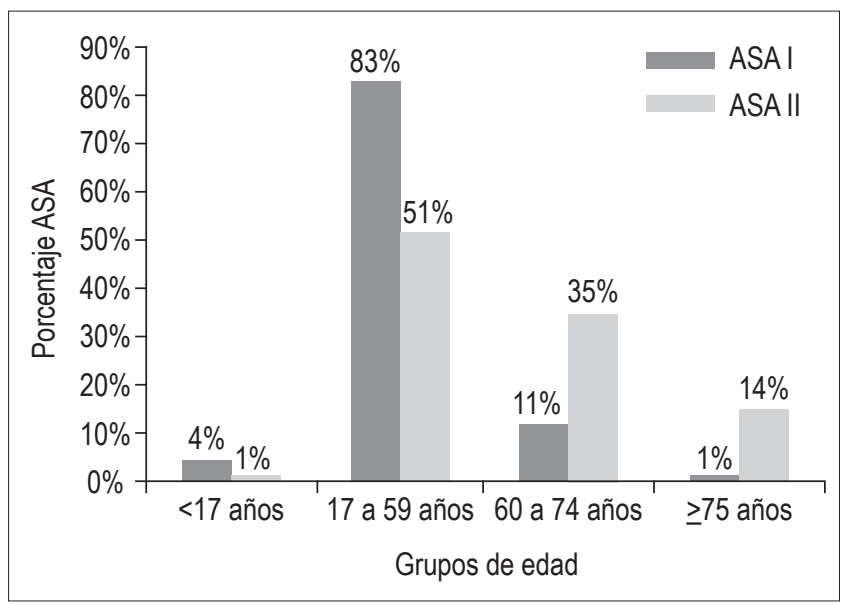

Figura 1. Distribución por grupos de edad y clasificación de la ASA.

Tabla 3. Distribución por grupos de edad y clasificación de la ASA

\begin{tabular}{lcccccc}
\hline Características generales & \multicolumn{9}{c}{ ASA } & \\
\cline { 2 - 7 } & \multicolumn{2}{c}{ I } & \multicolumn{2}{c}{ II } & \multicolumn{2}{c}{ Total } \\
\hline Grupos de edad & $\mathbf{N}$ & $\%$ & $\mathbf{N}$ & $\%$ & $\mathbf{n}$ & $\%$ \\
$\quad<17$ años & 35 & $4 \%$ & 2 & $1 \%$ & 37 & $3 \%$ \\
17 a 59 años & 657 & $83 \%$ & 180 & $51 \%$ & 837 & $73 \%$ \\
60 a 74 años & 90 & $11 \%$ & 123 & $35 \%$ & 213 & $19 \%$ \\
$\quad>75$ años & 11 & $1 \%$ & 50 & $14 \%$ & 61 & $5 \%$ \\
Total & 793 & $69 \%$ & 355 & $31 \%$ & & 1148 \\
\hline
\end{tabular}

En relación con las dosis por $\mathrm{kg}$ de peso, a los pacientes $<17$ años les fue administrada una dosis promedio de remifentanilo de $1,09 \mu \mathrm{g}(\mathrm{DE} \pm 0,06 \mu \mathrm{g})$, y entre los $17 \mathrm{y}$ 59 años, $0,98 \mu \mathrm{g}(\mathrm{DE} \pm 0,126 \mu \mathrm{g})$, siendo estos datos acordes con el promedio general de la dosis de $1 \mu \mathrm{g}$ por $\mathrm{kg}$ de peso. Se resalta que el promedio de la dosis en los pacientes mayores se disminuyó a 0,76 $\mu \mathrm{g}$ para pacientes entre los 60 y 74 años de edad $(\mathrm{DE} \pm 0,125 \mu \mathrm{g})$ y para pacientes $\geq 75$ años el promedio fue $0,71 \mu \mathrm{g}$ (DE 0,126 $\mu \mathrm{g})$.

Por su parte, a los pacientes $<17$ años les fue administrada una dosis por $\mathrm{kg}$ de peso promedio de propofol de $0,57 \pm 0,06 \mathrm{mg}$ y entre los 17 y 59 años, $0,49 \pm 0,06 \mathrm{mg}$; siendo estos datos acordes con el promedio general de la dosis por kilogramo de peso mencionado. En los pacientes entre los 60 y 74 años de edad la dosis fue de $0,39 \pm 0,06$ mg y para los $\geq 75$ años el promedio fue de $0,37 \pm 0,06 \mathrm{mg}$ (Figura 2, Tabla 4).

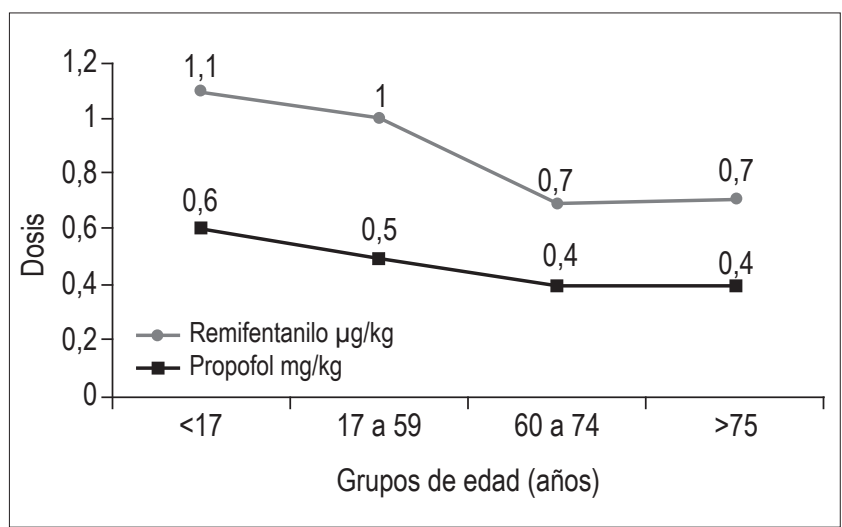

Figura 2. Dosis ideal por kg de peso y grupo de edad.

Respecto a los niveles de sedación logrados, el 94\% $(n=1084)$ estuvo en los ideales II y III. El 3\% ( $n=34)$ requirió estimulo vigoroso para mantener su ventilación (nivel IV) y solo el $2 \%(\mathrm{n}=28)$ llegó a nivel V de sedación, todos por hipoxemia que requirió asistencia ventilatoria con dispositivo de presión positiva, de los cuales solo 1 fue llevado a intubación orotraqueal (bajo relajación con succinilcolina) por presentar espasmo laríngeo (condición que, de acuerdo con la historia del paciente, ya había presentado espontáneamente con episodios de tos). No hubo en la serie eventos de hipotensión, anafilaxia, bradicardia o muerte, en especial por la rápida intervención de soporte ventilatorio en pacientes con niveles de sedación IV y V (Tabla 5).

La percepción de satisfacción del gastroenterólogo evidenció que, bajo sedación, la endoscopia fue un procedimiento fácil en el 94\% ( $\mathrm{n}=1084)$, que se relaciona con niveles de sedación II y III. Consideró que el procedimiento se pudo realizar en forma adecuada en el $3 \%(n=34)$, en el mismo número de pacientes con nivel de sedación IV. La percepción de procedimiento difícil se dio en el $3 \%(n=30)$, quienes al paso inicial del endoscopio se agitaron o intentaron retirarlo, o pujaban y tenían náuseas; en esta categoría también estuvieron los pacientes que no recuperaron la ventilación con el estímulo vigoroso y se debió pasar al apoyo de la asistencia ventilatoria dirigida por el anes-

Tabla 4. Dosis por kg de peso y por grupos de edad

\begin{tabular}{lcccc}
\hline & \multicolumn{4}{c}{ Dosis por kg de peso } \\
\hline Grupos de edad & $<17$ años & 17 a 59 años & 60 a 74 años & $>75$ años \\
Remifentanilo, media $\mu \mathrm{g} / \mathrm{kg} \pm \mathrm{DE}$ & $1,09 \pm 0,06$ & $0,98 \pm 0,126$ & $0,76 \pm 0,125$ & $0,71 \pm 0,126$ \\
Propofol, media $\mathrm{mg} / \mathrm{kg} \pm \mathrm{DE}$ & $0,57 \pm 0,06$ & $0,49 \pm 0,06$ & $0,39 \pm 0.06$ & $0,37 \pm 0,06$ \\
Total de pacientes: 1148 & & & & \\
\hline
\end{tabular}


tesiólogo. Una vez recuperada la ventilación espontánea, se pudo completar la endoscopia en todos los pacientes, incluida la paciente que requirió intubación (Tabla 6).

Tabla 5. Niveles de sedación logrados de acuerdo con la ASA

\begin{tabular}{lcccccc}
\hline \multirow{2}{*}{$\begin{array}{l}\text { Nivel de } \\
\text { sedación }\end{array}$} & \multicolumn{9}{c}{ ASA } \\
\cline { 2 - 7 } & \multicolumn{2}{c}{$\mathbf{I}$} & \multicolumn{2}{c}{ II } & \multicolumn{2}{c}{ Total } \\
\cline { 2 - 7 } & $\mathbf{n}$ & $\%$ & $\mathbf{n}$ & $\%$ & $\mathbf{n}$ & $\%$ \\
\hline I & 2 & $0 \%$ & 0 & $0 \%$ & 2 & $0 \%$ \\
II-III & 752 & $95 \%$ & 332 & $94 \%$ & 1084 & $94 \%$ \\
IV & 25 & $3 \%$ & 9 & $3 \%$ & 34 & $3 \%$ \\
V & 14 & $2 \%$ & 14 & $4 \%$ & 28 & $2 \%$ \\
\hline
\end{tabular}

Tabla 6. Satisfacción del endoscopista y relación con la ASA

\begin{tabular}{lcccccc}
\hline $\begin{array}{c}\text { Satisfacción del } \\
\text { endoscopista }\end{array}$ & \multicolumn{2}{c}{ ASA I } & \multicolumn{2}{c}{ ASA II } & \multicolumn{2}{c}{ Total } \\
\hline Adecuado & 25 & $3 \%$ & 9 & $3 \%$ & 34 & $3 \%$ \\
Fácil & 752 & $95 \%$ & 332 & $94 \%$ & 1084 & $94 \%$ \\
Difícil & 16 & $2 \%$ & 14 & $4 \%$ & 30 & $3 \%$ \\
Total & 793 & $69 \%$ & 355 & $31 \%$ & 1148 & $100 \%$ \\
\hline
\end{tabular}

Basado en el cálculo sobre el peso promedio de los pacientes de la serie $(66 \mathrm{~kg})$, en una mezcla de $0,5 \mathrm{mg} / \mathrm{kg}$ de propofol y de $1 \mu \mathrm{g} / \mathrm{kg}$ de remifentanilo; y teniendo en cuenta que, a octubre de 2016, $10 \mathrm{mg}$ de propofol costaron en pesos colombianos (COP) \$261 y $20 \mu \mathrm{g}$ de remifentanilo costaron $\$ 347,14$, el costo de los medicamentos fue de $\$ 861$ (en dólares a tasa de cambio de 1: 3000 , US $\$ 0,28$ ) y $\$ 1145$ (US $\$ 0,38$ ) respectivamente, para un valor total de \$2006 (US \$0,66) (Tabla 7).

Tabla 7. Promedio del costo de medicamentos en paciente de $66 \mathrm{~kg}$ a las dosis ideales descritas

\begin{tabular}{lcc}
\hline \multirow{2}{*}{ Medicamento } & \multicolumn{2}{c}{ Valor promedio por paciente } \\
\cline { 2 - 3 } & COP & US \\
\hline Remifentanilo & $\$ 1145$ & $\$ 0,38$ \\
Propofol & $\$ 846$ & $\$ 0,28$ \\
Total & $\$ 2006$ & $\$ 0,66$ \\
\hline
\end{tabular}

\section{DISCUSIÓN}

Diferentes estudios demuestran que al comparar la endoscopia sin o con sedación, esta última proporciona una mayor tolerancia para el paciente y una mejor aceptación para repetir la misma técnica realizada $(13,14)$. Es así que consideramos a la sedación como un componente integral dentro de los procedimientos endoscópicos modernos, pues el desarrollo de sus diferentes técnicas permite una mejor tolerancia, al reducir la ansiedad, el dolor y el recuerdo desagradable asociado con tiempos prolongados de intervención (por ejemplo en colangiopancreatografía retrógrada endoscópica [CPRE] o ultrasonografía endoscópica [UES]) como cuando hay que repetir con cierta periodicidad los exámenes para el seguimiento de diferentes patologías, como en el caso de pacientes con riesgo de cáncer $(15,16)$.

El esquema ideal de sedación apunta a tener un inicio rápido de acción sedante y analgésica, con facilidad del control del nivel de sedación deseado, con desaparición rápida del efecto sedante $y$, por tanto, rápida recuperación, todo dirigido a mantener un adecuado perfil de seguridad para el paciente (17).

\section{Seguridad}

El perfil de seguridad se basa en que el equipo de trabajo endoscópico tenga claramente definido el nivel de profundidad que se quiere alcanzar, entendida la sedación como un contínuo desde un nivel de ansiolisis hasta el nivel de anestesia general (contínuo no absolutamente controlable). El nivel buscado es determinado primero por el tipo de procedimiento a realizar, pero también por factores inherentes al paciente $(1,18)$. En el nivel ligero a moderado de sedación (sedación consciente en este trabajo) el paciente puede responder a órdenes verbales o táctiles suaves y simples para aumentar la frecuencia o profundidad de su respiración, suprimir su deglución o mantener inmóviles sus miembros (19), manteniendo su función cardiorrespiratoria y sus reflejos de protección, que suprime las náuseas y mejora la cooperación del paciente (4).

Por una parte, los riesgos asociados con la sedación exigen que el personal involucrado (anestesiólogos y no anestesiólogos) tenga formación y conocimiento en la detección y reversión de estados de sedación no deseados o innecesarios para el tipo de procedimiento que se esté realizando, con formación en soporte vital básica y avanzada y, en especial, que el médico encargado de la sedación esté exclusivamente para su administración y monitorización (16). Otros elementos de seguridad tienen que ver con instalaciones y accesorios (fuente de oxígeno, succionador independiente al de la endoscopia, equipo básico y avanzado para el manejo de la vía respiratoria, sondas de aspiración de secreciones, mascarilla, dispositivo de presión positiva, laringoscopio, tubos endotraqueales); medicamentos para reversión de los fármacos usados y para el manejo de alergias y de reanimación cardiopulmonar avanzada; equipos de monitorización para presión arterial (PA), frecuencia cardíaca (FR), pulsoximetría, electrocardiografía y eventualmente capnografia; sala de recuperación con monitorización, fuente de oxígeno y de succión; y personal específico encargado de esta área $(20,21)$. 
Por otra parte y a pesar de la normatividad que obliga a que el propofol solo debe ser administrado por anestesiólogos, existen múltiples revisiones y consensos que apoyan su seguridad y eficiencia al ser usados por médicos y enfermeras adecuadamente capacitados $(22,23)$. Por ejemplo, en una serie tan grande como de 646080 pacientes en los que solo se requirieron 11 intubaciones traqueales, sin ningún daño neurológico permanente y con 4 muertes de pacientes con comorbilidades importantes y no estrictamente atribuibles a la sedación (24). Otros autores han concluido que la sedación tradicional con benzodiacepinas y opiáceos obtiene un índice menor de mortalidad que cuando se usa propofol, y un índice similar al que se presenta en anestesia general realizada por anestesiólogos (17). También concluyen que la sedación administrada por no anestesiólogos puede incluso tener mayores niveles de seguridad en pacientes de ASA > III, siempre y cuando se dediquen en forma única y exclusiva a administrar y controlar la sedación (25-27).

Con todo, institucionalmente se optó por la participación del anestesiólogo (incluido el incremento de costo que ello implica) por la seguridad que también aporta, siempre y cuando este procure un nivel de sedación consciente II-III, ya que la eventual confianza en sus conocimientos de reanimación, en los medicamentos, en las dosis o en la velocidad de inyección puede llevar al paciente a niveles más profundos de sedación, lo que genera iguales o mayores riesgos que una sedación administrada por no anestesiólogos.

Es importante, entonces, conocer las diferentes técnicas de sedación y seleccionar la más adecuada de acuerdo con la experiencia del grupo de endoscopia, del procedimiento a realizar, de la clasificación ASA, de las expectativas del paciente, de los medicamentos disponibles (28) y de las regulaciones institucionales, locales y nacionales (para el momento de esta serie 2013-2014 no había obligatoriedad de que el administrador de la sedación fuera un profesional diferente al endoscopista, hecho que ulteriormente quedó decretado en Colombia en el año 2014) (29).

\section{Eficiencia}

En el ámbito de la endoscopia digestiva, realizar un análisis de la eficiencia o costo-efectividad es muy difícil puesto que en sus cálculos intervienen múltiples factores que hacen que un esquema o modelo pueda ser enormemente eficiente en un escenario, pero un fracaso total en otro.

El esquema aquí presentado ha sido exitoso para nuestra institución, pero su implementación inicial ha generado un incremento en los costos en 4 aspectos principales: costo de medicamentos (propofol, remifentanilo, naloxona, medicamentos de carro de paro, oxígeno, entre otros); material desechable (jeringas, yelcos, tapones, tubos endotraqueales, cánulas de succión y de oxígeno); material no desechable (sistemas de monitorización); y personal adicional (anestesiólogos, enfermeras) con sus respectivas capacitaciones.

Diferentes publicaciones (17) han demostrado que la sedación aporta a una realización de exámenes más completos y de mejor calidad y a disminuir las reprogramaciones, eficiencia que compensa parcialmente los gastos expuestos arriba; a su vez, la mejor tolerancia y satisfacción de los pacientes genera a largo plazo una preferencia social respecto a la atención recibida.

Otros parámetros que se han utilizado como medidores de eficiencia de los sistemas de sedación son el tiempo de recuperación (desde el final del procedimiento hasta lograr escala de Aldrete mínimo de 10), inducción de la sedación (tiempo desde la primera inyección hasta el comienzo de la sedación efectiva), calidad de la sedación (evaluada por los endoscopistas, las enfermeras y los pacientes, con medición al final del procedimiento), y las complicaciones relacionadas con el procedimiento (33).

En la presente serie, la calidad de la sedación en la endoscopia fue medida de acuerdo con la percepción del endoscopista, utilizando una escala de 3 niveles para el procedimiento en general (fácil, adecuado y difícil) que hacen referencia a lo que sintió el operador frente a la cooperación del paciente, ausencia de náuseas o arcadas, mantenimiento adecuado de la ventilación sin necesidad de intervención, ausencia de dolor, aceptable nivel de amnesia con expresión de satisfacción del paciente.

La serie muestra que para el endoscopista el procedimiento global fue fácil en el $94 \%$ (asociado con niveles de sedación II y III), adecuado en el 3\% (para niveles de sedación I y IV, en los que se requirió dosis adicional de medicamentos o estímulo vigoroso, que ambos interrumpían momentáneamente la continuidad de la endoscopia, sin necesidad de retirar el endoscopio); y difícil en el 3\% (asociado con niveles de sedación $\mathrm{V}$ que exigieron interrupción del examen, asistencia ventilatoria con dispositivo de presión positiva -en un solo caso con intubación orotraqueal- y una vez recuperada la ventilación espontánea con reintroducción del endoscopio) (12).

\section{Los medicamentos (propofol y remifentanil)}

En nuestra experiencia acumulada no publicada en más de 60000 endoscopias bajo sedación, utilizamos diferentes técnicas de inyección (con bomba de infusión, con goteo continuo, en bolos); diferentes medicamentos en monosedación (diazepam, midazolam, fentanilo o propofol). Pero en 2004 dimos el paso a la sedación balanceada con propofol, originalmente descrita por Cohen y colaboradores para sedación moderada (8), en la que se combinan la sedación balanceada en pequeñas dosis incrementales de propofol con dosis pequeñas de benzodiacepinas y opioides, que 
incluso es administrada inicialmente por un médico no anestesiólogo (30). La acción sinérgica de los medicamentos en sedación consciente permite reducir la dosis total del propofol (frente a su dosis requerida en monosedación) y reduce el riesgo de complicaciones cardiovasculares relacionadas con su uso $(31,32)$. A su vez, el uso combinado con opiáceos o benzodiacepinas apunta a obtener una adecuada somnolencia, amnesia y analgesia (33).

Es de mencionar que el advenimiento de propofol nos permitió cambiar el esquema previo de sedación balanceada, de benzodiacepina-opioide (midazolam con remifentanilo), toda vez que las depresiones respiratorias eran muy frecuentes y su tiempo de duración era más prolongado, experiencia ulteriormente descrita en otros estudios en cuyos procedimientos con dicha combinación se llegaba a sedación profunda en hasta el $68 \%$ de los pacientes (85\% en ultrasonido endoscópico y CPRE, $60 \%$ en endoscopias digestivas altas y $45 \%$ en colonoscopia) $(34,35)$. La ventaja de este esquema era que el afecto amnésico potente de la benzodiacepina hacía que no hubiera recordación desagradable del paciente, tanto si llegaba solo a un nivel I de sedación como si hubiera requerido estímulos vigorosos o maniobras de asistencia ventilatoria.

Las ventajas del propofol (2,6-diisopropilfenol) provienen de ser un agente hipnótico de muy corta duración con un comienzo de acción rápido (usualmente entre 30 y 60 segundos) y un tiempo de recuperación corto; (entre 4 y 8 minutos); con efecto analgésico mínimo, muy buen nivel de amnesia, degradación en el primer paso hepático (terminando en forma rápida sus efectos) y con rápido retorno de la conciencia. Es altamente lipofílico y cruza rápidamente la barrera hematoencefálica; está contraindicado en pacientes alérgicos al huevo o a la soya, puesto que en su presentación más común es una emulsión que contiene aceite de soya al $10 \%$, glicerol al 2,25\%, y lecitina de huevo al $1,2 \%$, aunque parece estar apareciendo evidencia que no valida esta prevención. Es un medicamento de categoría B en el embarazo y debe usarse con precaución en la lactancia (4).

Estas características han hecho que su uso mundial se haya aumentado en la última década, pues da una seguridad comparable con la sedación tradicional con benzodiacepinas y opiáceos $(2,14,36,37)$; y por su facilidad de alcanzar niveles de sedación más profunda (que dependen de la dosis) es una alternativa real para procedimientos tanto endoscópicos de corta duración como para los prolongados (CPRE o UES) (38). Sin embargo, esa potencialidad de inducir mayor profundidad de sedación junto con la ausencia de un antagonista específico ha propiciado la normatividad descrita arriba frente a la restricción de su uso (4, 17) generando una percepción frente a los riesgos que en EEUU ha hecho que los endoscopistas quieran su uso, pero sean reacios a hacerlo (38).
Por su parte, el remifentanilo es un opioide de acción ultrarrápida (inicio a los 30 segundos) y corta duración de acción con vida media de 8 a 10 minutos, por lo que es predecible su terminación de acción. Su potencia analgésica es similar al fentanilo y 20 a 30 veces más potente que el alfentanilo, pero su duración de acción es mucho más corta que los 2, pues es rápidamente metabolizado por las estearasas no específicas de la sangre y de otros tejidos (39). Su uso en altas dosis produce pérdida de consciencia y se asocia con rigidez muscular y tórax en leño (40). En ancianos, su comienzo de acción y aumento de vida media pueden prolongarse, por lo que debe reducirse su dosis hasta el 50\%. En obesos la dosis debe calcularse con base en el índice de masa corporal (IMC). Sus efectos a nivel cardiovascular pueden ser hipotensión y bradicardia, y al asociarse con propofol puede reducir la PA en un $17 \%-23 \%$, pero en general proporciona una buena estabilidad hemodinámica. La depresión respiratoria que puede producir es dependiente de la dosis. Su aclaramiento no está alterado en disfunción hepática o de colinesterasa plasmática, y su principal metabolito está elevado en pacientes con insuficiencia renal, pero no tiene influencia clínica pues es escasa la potencia del mismo. Además de usarse como analgésico durante la inducción anestésica, es una alternativa en la sedación balanceada con propofol. El aumento de su dosis puede causar tórax en leño y cierre glótico y se presentan cuando se administra rápidamente, de allí que se sugiera la administración en titulación lenta. No se recomienda su uso en embarazadas, durante la lactancia ni en menores de 2 años. Hasta en el $9 \%$ de los pacientes puede haber rigidez muscular, pero esta se reduce a $1 \%$ cuando se usa junto con un hipnótico potente o con un relajante muscular, esto en el escenario de la anestesia general o en el uso de infusión continua $(34,39,41,42)$.

Como se menciona antes, el propofol no tiene un antagonista específico disponible en este momento. Para el remifentanilo, el antagonista competitivo es la naloxona, que debe administrarse vía endovenosa para revertir efectos adversos descritos arriba, pero teniendo en cuenta que puede producir liberación de catecolaminas, taquiarritmias e incluso muerte súbita en pacientes con cardiopatía subyacente, o síndromes de abstinencia en pacientes farmacodependientes de narcóticos (4). En esta serie de pacientes no tuvimos la necesidad de utilizar reversión farmacológica, toda vez que el evento más frecuente fue la depresión respiratoria en nivel de sedación $\mathrm{V}$, que solo requirió adecuada y paciente asistencia ventilatoria en períodos no mayores a 2 minutos.

\section{Sedación balanceada}

Actualmente hay suficiente evidencia que demuestra cómo el propofol solo o en combinación con un opiáceo es el fár- 
maco de elección para procedimientos endoscópicos y que en el esquema de sedación balanceada es posible reducir su dosis consiguiendo el mismo efecto hipnótico. Igualmente, en sedación balanceada el opioide más favorable por su perfil farmacocinético y farmacodinámico es el remifentanilo, dada su rápida eliminación mencionada previamente, pero su potencia da un margen terapéutico estrecho que obliga a su administración controlada por parte de un anestesiólogo (17).

Diferentes estudios de procedimientos endoscópicos pancreatobiliares donde se compara el propofol como monosedación o en esquemas balanceados, reportan que la dosis para inducción en bolo intravenoso (IV) es de 0,5 $\mathrm{mg}$ a $1 \mathrm{mg}$ por $\mathrm{kg}$ de peso en bolo de $30 \mathrm{~s}$; seguido por dosis repetidas de 10 a $20 \mathrm{mg}$ (o $0,25 \mathrm{mg} / \mathrm{kg}$ ), para mantener una adecuada sedación de acuerdo con el nivel deseado y con el perfil de riesgo del paciente, con dosis medias totales de $185 \mathrm{mg}(33,34)$, que se reducen a $106 \mathrm{mg}$ cuando se balancea el propofol con midazolam o meperidina (7); o a $117 \mathrm{mg}$ hasta $175 \mathrm{mg}$ en el esquema balanceado con remifentanilo-ketamina (34).

Dado que se conoce que los anestésicos IV, tales como hipnóticos, opioides o benzodiacepinas, se combinan sinérgicamente (43) y se asocian para potenciarse (44), con el balanceo se busca lograr sus efectos deseados con las dosis más bajas posibles, así: se ha descrito que la asociación de remifentanilo con propofol en exámenes endoscópicos y en punción lumbar de pacientes pediátricos permite una recuperación extremadamente rápida con muy corta duración de sus efectos (45); también, que con dosis de remifentanilo de $0,3 \mu \mathrm{g} / \mathrm{kg}$ y de propofol de $1 \mathrm{~mL} / 3 \mathrm{~s}$ hay una reducción del dolor en el sitio de inyección del propofol de hasta el 11\%; con una reducción en la dosis total de propofol de 2,07 mg/kg (en monosedación) frente a 1,19 mg/ $\mathrm{kg}$ (rangos entre 0,51 a 1,91 mg/kg) cuando se utilizó en esquema balanceado con remifentanilo a la dosis descrita arriba de $0,3 \mu \mathrm{g} / \mathrm{kg}(46)$.

De acuerdo con lo anterior, el esquema de sedación balanceada con propofol y remifentanilo para sedación en endoscopia digestiva diagnóstica cumple con características ideales medicamentosas de rápida conexión con el sitio de efecto, reducida acumulación en el cuerpo, rápida eliminación y efectos farmacodinámicos, como hipnosis temprana, sedación moderada a profunda, rápido y eficiente control de respuestas autonómicas, rápido retorno de la consciencia y, por tanto, mayor predictibilidad de sus efectos, lo que da un mayor margen de seguridad y de prevención de efectos adversos (46).

La selección de remifentanilo frente a fentanilo o alfentanilo es que con los 2 últimos la duración de su efecto puede extenderse demasiado en el período de recuperación, hecho no deseable ni eficiente en procedimientos cortos como las endoscopias digestivas diagnósticas (47).
Resultados por Hayes (45) en 2008 mostraron que en la combinación propofol-remifentanilo, al incrementar la dosis de remifentanilo a $1,5 \mu \mathrm{g} / \mathrm{kg}$ y disminuir la dosis de propofol a $2 \mathrm{mg} / \mathrm{kg}$, se incrementaba el tiempo de duración de apnea, pero se reducía el tiempo de recuperación. A su vez, al reducir el remifentanilo a $0,5 \mu \mathrm{g} / \mathrm{kg}$ e incrementar el propofol a $4 \mathrm{mg} / \mathrm{kg}$, el tiempo de apnea se reducía, pero el período de recuperación se incrementaba. Nuestro esquema optó por la primera alternativa, es decir, mayores dosis de remifentanilo que de propofol; con lo cual logramos tiempos de recuperación más cortos con mínima proporción de pacientes que requirieron estímulo vigoroso o asistencia ventilatoria ( $3 \%$ nivel de sedación IV y $2 \%$ nivel de sedación V). De cualquier modo, siempre tuvimos claro y estuvimos atentos del potencial efecto depresivo ventilatorio de ambos medicamentos interactuantes $y$, basados en el principio de la curva de interacción asimétrica propuesta por Fidler (48), decidimos utilizar las más bajas dosis necesarias de remifentanilo y propofol (Figura 2) de acuerdo con nuestra experiencia en ese momento y que, como se puede apreciar, fueron seguras para los pacientes.

Un efecto no cuantificado en esta serie, pero sí percibido y descrito en estudios, fue la disminución del dolor en el sitio de venopunción con propofol. Aunque la presentación más común de propofol es en emulsión, su aplicación causa dolor e incluso flebitis, en especial si la inyección es por venas periféricas pequeñas. La administración de bajas dosis de remifentanilo unos segundos antes disminuye efectivamente el dolor (49-51).

\section{Limitaciones de la serie}

Como en toda serie de casos, no hay un grupo de comparación de resultados frente a otras dosis de los mismos medicamentos, aunque es claro que las edades extremas (jóvenes y tercera edad) requieren dosis diferenciadas por una diferencia evidente en la sensibilidad de los medicamentos. Tampoco están incluidos los tiempos de recuperación y egreso, ni la medición de la satisfacción del paciente, obviada por resultados nuestros (no publicados) de las encuestas telefónicas de seguimiento a pacientes que nos reportan niveles de satisfacción $>95 \%$ en todo el proceso de atención, incluida la sedación.

\section{CONCLUSIONES}

1. La sedación balanceada es un esquema seguro cuando es administrado por un médico diferente al operador; para la presente serie, con la obligatoriedad de su administración por un anestesiólogo dada la restricción para el uso que cobija al propofol. 
2. La potenciación que ejercen los medicamentos combinados permite reducir en forma significativa las dosis necesarias de estos eventualmente a niveles menores a los encontrados en esta serie.

3. La reducción de las dosis facilita (aunque no asegura) obtener niveles de sedación consciente (II y III) para procedimientos de endoscopia alta diagnóstica.

4. Los costos totales de los medicamentos en sedación balanceada son muy bajos, pero se debe tener en cuenta que los costos globales del montaje de un sistema de atención bajo sedación se incrementan en una forma muy significativa, en especial por los equipos de monitorización y por los honorarios del anestesiólogo. Sin embargo, a mediano plazo los resultados generales pueden ser altamente eficientes.

5. A pesar de la seguridad del esquema presentado, no se debe olvidar que la sedación puede ser un contínuo desde el estado de alerta hasta la anestesia general, por lo que se deben extremar todas las medidas de monitorización y de capacitación humana, con los cuales se asegure en especial la experticia en el rescate de la vía aérea, mediante la adherencia a listas de chequeo y a modelos de entrenamiento teórico y práctico clínico en un número mínimo de pacientes, para el salvamento y recuperación de niveles de sedación profundos no esperados.

\section{REFERENCIAS}

1. Knape JT, Adriaensen H, van Aken H, et al. Guidelines for sedation and/or analgesia by non-anaesthesiology doctors. Eur J Anaesthesiol. 2007;24(7):563-7. Doi: https://doi. org/10.1017/S0265021506002092

2. Cohen LB, Delegge MH, Aisenberg J, et al. AGA Institute rewiew of endoscopic sedation. Gastroenterology 2007; 133(2):675701. Doi: https://doi.org/10.1053/j.gastro.2007.06.002

3. American Society of Anesthesiologists Task Force on Sedation and Analgesia by Non-Anesthesiologists. Practice guidelines for sedation and analgesia by non-anesthesiologists. Anesthesiology. 2002;96(4):1004-17. Doi: https:// doi.org/10.1097/00000542-200204000-00031

4. Lightdale JR. Preparación y sedación del paciente para endoscopia. En: Classen M, Tytgat GNJ, Lightdale CJ, et al. (editores). Endoscopia gastroenterológica. 2.a edición. Venezuela: Amolca; 2012. p. 57-65.

5. MaoW,WeiXQ, TaoJ, et al. The safety of combined sedation whit propofol plus fentanyl for endoscopy screening and endoscopic variceal ligation in cirrhotic patients. J Dig Dis. 2014;15(3):12430. Doi: https://doi.org/10.1111/1751-2980.12115

6. Jamieson J. Anesthesia and sedation in the endoscopy suite? (Influences and opinions). Curr Opin Anesthesiol. 1999; 12(4):417-23.Doi:https://doi.org/10.1097/00001503199908000-00004

7. Lee KC, Lee S-H, Chung I-H, et al. Balanced propofol sedation for therapeutic GI endoscopic procedures: a prospective, randomized study. Gastrointest Endosc. 2011;73(2):206-14. Doi: https://doi.org/10.1016/j.gie.2010.09.035

8. Cohen LB, Hightower CD, Wood DA, et al. Moderate level sedation during endoscopy: a prospective study using low-dose propofol, meperidine/fentanyl, and midazolam. Gastrointest Endosc. 2004;59(7):795-803. Doi: https:// doi.org/10.1016/S0016-5107(04)00349-9 https://doi. org/10.1016/S0016-5107(04)00628-5

9. Keats AS. The ASA classification of physical status-a recapitulation. Anesthesiology. 1978;49(4):233-6. Doi: https:// doi.org/10.1097/00000542-197810000-00001

10. Yao K. The endoscopic diagnosis of early gastric cancer. Ann Gastroenterol. 2013;26(1):11-22.

11. Lee JY, Kim N. Diagnosis of Helicobacter pylori by invasive test: histology. Ann Transl Med. 2015;3(1):10.

12. Sachdeva A, Bhalla A, Sood A, et al. The effect of sedation during upper gastrointestinal endoscopy. Saudi J Gastroenterol. 2010;16(4):280-4. Doi: https://doi.org/10.4103/13193767.70616

13. Kenneth R, Laine L. A systematic review and meta-analysis of randomized, controlled trials of moderate sedation for routine endoscopic procedures. Gastrointest Endosc. 2008;67(6):910-23. Doi: https://doi.org/10.1016/j. gie.2007.12.046

14. Abraham NS, Fallone CA, Mayrand S, et al. Sedation versus no sedation in the performance of diagnostic upper gastrointestinal endoscopy: a Canadian randomized controlled costoutcome study. Am J Gastroenterol. 2004;99(9):1692-9. Doi: https://doi.org/10.1111/j.1572-0241.2004.40157.x

15. DeLegge $\mathrm{MH}$. When to call the anesthesiologist for assistance whit sedation. Gastrointest Endosc. 2011;74(6):13779. Doi: https://doi.org/10.1016/j.gie.2011.10.005

16. American Society of Anesthesiologists. Task Force on Sedation and Analgesia by Non-Anesthesiologists. Practice Guidelines for sedation and Analgesia. An Updated. Anesthesiology. 2002; 96: 1004-17. Doi: https://doi. org/10.1097/00000542-200204000-00031

17. Gonzalez-Huix F, Giné J, Loras C, et al. Documento de posicionamiento de la sociedad catalana de digestología sobre la sedación en endoscopia digestiva. Gastroenterol Hepatol. 2012;35(7):496-511. Doi: https://doi.org/10.1016/j.gastrohep.2012.03.009

18. Faigel DO, Baron TH, Goldstein JL, et al. Guidelines for the use of deep sedation and anesthesia for GI endoscopy. Gastrointest Endosc. 2002;56(5):613-7.

19. Chutkan R, Cohen J, Abedi M, et al. Training guideline for use of propofol in gastrointestinal endoscopy. Gastrointest Endosc. 2004;60(2):167-72. Doi: https://doi. org/10.1016/S0016-5107(04)01699-2

20. Dumonceau JM, Riphaus A, Aparicio JR, et al. European Society of Gastrointestinal Endoscopy, European Society of Gastroenterology and Endoscopy Nurses and Associates, and the European Society of Anaesthesiology Guideline: Non-anesthesiologist administration of propofol for GI endoscopy. Endoscopy. 2010;42(11):960-74. Doi: https:// doi.org/10.1055/s-0030-1255728 
21. Cohen LB, Delegge MH, Aisenberg J, et al. AGA Institute review of endoscopic sedation. Gastroenterology. 2007;133(2):675701. Doi: https://doi.org/10.1053/j.gastro.2007.06.002

22. Cohen APT, Cohen LB, Ladas SD, et al. Sedation in digestive endoscopy: the Athens international position statements. Aliment Pharmacol Ther. 2010;32(3):425-42. Doi: https://doi.org/10.1111/j.1365-2036.2010.04352.x

23. Ellet ML. A literature review of the safety and efficacy of using propofol for sedation in endoscopy. Gastroenterol Nurs. 2010;33(2):111-7. Doi: https://doi.org/10.1097/ SGA.0b013e3181d601fb

24. Rex DK, Deenadayalu VP, Eid E, et al. Endoscopist-directed administration of propofol: a worldwide safety experience. Gastroenterology. 2009;137(4):1229-37. Doi: https://doi. org/10.1053/j.gastro.2009.06.042

25. Martinez JF, Aparicio JR, Compañy L, et al. Safety of continuous propofol sedation for endoscopic procedures in elderly patients. Rev Esp Enferm Dig. 2011;103(2):76-82. Doi: https://doi.org/10.4321/S1130-01082011000200005

26. García-Suárez C, López-Rosés L, Olivencia $P$, et al. Sedation whit propofol controlled by endoscopists during percutaneous endoscopic gastrostomy. Rev Esp Enferm Dig. 2010;102(4):249-56. Doi: https://doi.org/10.4321/S113001082010000400005

27. Heuss LT, Schnieper P, Drewe J, et al. Safety of propofol for conscious sedation during endoscopic procedures in high-risk patients-a prospective, controlled study. Am J Gastroenterol. 2003;98(8):1751-7. Doi: https:// doi.org/10.1016/S0002-9270(03)00447-7 https://doi. org/10.1111/j.1572-0241.2003.07596.x

28. Lichtenstein DR, Jagannath S, Baron TH, et al. Sedation and anesthesia in GI endoscopy. Standards of Practice Committee of the American Society for Gastrointestinal Endoscopy. Gastrointest Endosc. 2008;68(5):815-26. Doi: https://doi.org/10.1016/j.gie.2008.09.029

29. Ministerio de Salud y Protección Social [Internet]. Resolución número 00002003 de 2014. Bogotá: Ministerio de Salud; 2014 [actualizado el 28 de mayo de 2014; acceso el 3 de septiembre de 2016]. Disponible en: https://www. minsalud.gov.co/Normatividad_Nuevo/Resolución\%20 2003\%20de\%202014.pdf.

30. Vargo JJ, Cohen LB, Rex DK, et al. Position statement: non anesthesiologist administration of propofol for GI endoscopy. Gastrointest Endosc. 2009;70:1053-9. Doi: https:// doi.org/10.1016/j.gie.2009.07.020

31. Ong WC, Santosh D, Lakhtakia S, et al. A randomized controlled trial on use of propofol alone versus propofol with midazolam, ketamine and pentazocine "sedate-analgesic cocktail" for sedation during ERCP. Endoscopy. 2007;39(9):807-12. Doi: https://doi.org/10.1055/s-2007-966725

32. Paspatis GA, Manolaraki MM, Vardas E, et al. Deep sedation for endoscopic retrograde cholangiopancreatography: intravenous propofol alone versus intravenous propofol whit oral midazolam premedication. Endoscopy. 2008;40(4):308-13. Doi: https://doi.org/10.1055/s-2007-995346
33. Lee TH, Lee CK, Park S-H, et al. Balanced propofol sedation versus propofol monosedation in therapeutic pancreaticobiliary endoscopic procedures. Dig Dis Sci. 2012;57(8):211321. Doi: https://doi.org/10.1007/s10620-012-2234-0

34. Fabbri LP, Nucera M, Marsili M, et al. Ketamine, propofol and low dose remifentanil versus propofol and remifentanil for ERCP outside the operating room; Is ketamine not only a "rescue drug"? Med Sci Monit. 2012;18(9): CR575-80. Doi: https://doi.org/10.12659/MSM.883354

35. Trummel J. Sedation for gastrointestinal endoscopy: the changing landscape. Curr Opin Anaesthesiol. 2007;20(4):359-64. Doi: https://doi.org/10.1097/ACO.0b013e32827ab467

36. McQuaid KR, Laine L.A systematic review and meta-analysis of randomized, controlled trials of moderate sedation for routine endoscopic procedures. Gastrointest Endosc, 2008;67(6):91023. Doi: https://doi.org/10.1016/j.gie.2007.12.046

37. Vargo JJ, Zuccaro Jr G, Dumot JA, et al. gastroenterologistadministered propofol versus meperidine and midazolam for advanced upper endoscopy: a retrospective, randomized trial. Gastroenterology. 2002;123(1):8-16. Doi: https:// doi.org/10.1053/gast.2002.34232

38. Cohen LB, Wecsler JS, Gaetano JN, et al. Endoscopic sedation in the United States: results from a nationwide survey. Am J Gastroenterol 2006;101(5):967-74. Doi: https://doi. org/10.1111/j.1572-0241.2006.00500.x

39. Anesnet [Internet]. La agenda del anestesiólogo. Madrid: Universidad Autónoma de Madrid; 1997 [actualizado 27 de mayo de 2010; acceso el 8 de septiembre de 2016]. Disponible en: https://www.uam.es/departamentos/medicina/anesnet/agenda/indice.htm.

40. Muñoz L, Arévalo JJ, Reyes LE, et al. Remifentanilo versus propofol con infusión controlada a objetivo en sitio efecto para la sedación de pacientes durante procedimientos endoscópicos gastrointestinales: ensayo clínico controlado aleatorizado. Rev Colomb Anestesiol. 2013;41(2):114-9. Doi: https://doi.org/10.1016/j.rca.2012.11.001

41. Rosow C. Remifentanil: a unique opioid analgesic. Anaesthesiology. 1993;79(5):875-6.

42. Medina HJ, Galvin EM, Dirckx M et al. Remifentanil as a single drug for extracorporeal shock wave lithotripsy: a comparison of infusion doses in term of analgesic potency and side effects. Anesth Analog. 2005;101(2):365-70. Doi: https://doi.org/10.1213/01.ANE.0000159379.54705.84

43. Minto CF, Schnider TW, Short TG, et al. Response surface model for anesthetic drug interactions. Anesthesiology. 2000;92(6):1603-16.Doi: https://doi.org/10.1097/00000542200006000-00017

44. Duarte NMC, Pires OC, Nunes CEL, et al. Anestesia venosa total. Sociedade Brasileira de Anestesiologia. 2011;73-82.

45. Hayes JA, Lopez AV, Pehora CM, et al. Coadministration of propofol and remifentanil for lumbar puncture in children. Anesthesiology. 2008;109(4):613-8. Doi: https://doi. org/10.1097/ALN.0b013e31818629f3

46. Uliana GN, Milla Tambara E, Pedroso Baretta GA. Use of remifentanil to reduce propofol injection pain and the requi- 
red propofol dose in upper digestive tract endoscopy diagnostic tests. Rev Bras Anestesiol. 2015;65(6):437-44. Doi: https://doi.org/10.1016/j.bjan.2015.08.002

47. Egan TD, Kern SE, Muir KT, et al. Remifentanil by bolus injection: a safety, pharmacokinetic, and age effect investigation in human volunteers. Br J Anaesth. 2004;92(3):335-43. Doi: https://doi.org/10.1093/bja/aeh075

48. Fidler M, Kern SE. Flexible interaction model for complex interactions of multiple anesthetics. Anesthesiology. 2006;105(2):286-96.Doi: https://doi.org/10.1097/00000542200608000-00011

49. Jeong CW, Lee SH, Ju J, et al. The effect of priming injection of different doses of remifentanil on injection pain of microemulsion propofol premixed with lidocaine. Korean J Anesthesiol. 2011;60(2):78-82. Doi: https://doi. org/10.4097/kjae.2011.60.2.78

50. Lee JR, Jung CW, Lee YW. Reduction of pain during induction with target-controlled propofol and remifentanil. Br J Anaesth. 2007;99(6):876-80. Doi: https://doi. org/10.1093/bja/aem293

51. Lee JY, Yang HJ, Choi SH, et al. The optimal effect-site concentration of remifentanil to attenuate the pain caused by propofol. Korean J Anesthesiol. 2012; 63(2):108-12. Doi: https://doi.org/10.4097/kjae.2012.63.2.108 